\title{
Role of New Hydrophilic Surfaces on Early Success Rate and Implant Stability: 1-Year Post-loading Results of a Multicenter, Split-Mouth, Randomized Controlled Trial
}

\author{
Marco Tallarico ${ }^{1}$ Nicola Baldini ${ }^{2}$ Fulvio Gatti ${ }^{3}$ Matteo Martinolli ${ }^{4}$ Erta Xhanari ${ }^{1}$ \\ Silvio Mario Meloni ${ }^{5}$ Cervino Gabriele ${ }^{6} \quad$ Lumbau Aurea Immacolata ${ }^{5}$
}

\footnotetext{
${ }^{1}$ School of Dentistry, University of Sassari, Sassari, Italy

${ }^{2}$ Department of Periodontics and Implantology,

University of Siena, Siena, Italy

${ }^{3}$ Department of Biomedical, Surgical and Dental Sciences,

Unit of Oral Surgery, San Carlo and San Paolo Hospital,

University of Milan, Milan, Italy

4Private Practice in Porto Viro, Porto Viro, Italy

${ }^{5}$ Medical Surgical and Experimental Science Department, University of Sassari, Sassari, Italy

${ }^{6}$ Department of BIOMORF, School of Dentistry,

University of Messina, Messina, Italy
}

\author{
Address for correspondence Silvio Mario Meloni, DDS, MSc, PhD \\ Medical Surgical and Experimental Science Department, \\ University of Sassari, Viale San Pietro 43 B, 07100 Sassari, Italy \\ (e-mail: melonisilviomario@yahoo.it).
}

Eur J Dent:2021;5:1-7

\begin{abstract}
Keywords

- dental implants

- implant surface

- osseointegration

- bone quality

Objective To compare early implant failure and implant stability of one-stage Hiossen ET III implants with its new hydrophilic (NH) surface, compared with Hiossen ET III implants with the sandblasted and acid-etched (SA) surface at 1-year follow-up. Materials and Methods This study was designed as a split-mouth, multicenter randomized controlled trial aimed to compare SA surface implants (SA group) and $\mathrm{NH}$ surface, (NH group). Outcomes were implant and prosthetic survival rates, complications, the insertion torque at implant placement, and implant stability quotient (ISQ) values.

Results Twenty-nine patients (mean age $59.9 \pm 11.3$ years) were treated and followed up to 1 year after loading. No patient dropped out. Fifty-eight implants (29 SA group and $29 \mathrm{NH}$ group) were placed. No implants or prostheses failed and no complications were experienced during follow-up. The mean insertion torque was $40.5 \pm 3.23(38.17-41.83) \mathrm{Ncm}$ in the SA group and $40.48 \pm 3.49(38.02-41.98) \mathrm{Ncm}$ in the $\mathrm{NH}$ group $(p=0.981)$. There was a statistically significant difference at the second week (T2) with higher values in the NH group $(p=0.041)$. Similar results were found in the maxilla $(p=0.045)$, but not in the mandible $(p=0.362)$. A positive correlation was found between initial insertion torque and ISQ with higher value in the $\mathrm{NH}$ group (0.73 vs. 0.66$)$.

Conclusions NH implants are a viable alternative to SA surface, as they seem to avoid the ISQ drop during the bone remodeling phase.
\end{abstract}

DOI https://doi.org/ $10.1055 / \mathrm{s}-0040-1713952$ ISSN 1305-7456. (c) 2020. European Journal of Dentistry.

This is an open access article published by Thieme under the terms of the Creative Commons Attribution License, permitting unrestricted use, distribution, and reproduction so long as the original work is properly cited. (https://creativecommons. org/licenses/by/4.0/)

Thieme Medical and Scientific Publishers Pvt. Ltd., A-12, 2nd Floor, Sector 2, Noida-201301 UP, India 


\section{Introduction}

Modern dentist has an excellent solution to solve patient's edentulism using dental implants. It has been shown that dental implants have long-term successful outcomes, representing a viable option for clinicians to rehabilitate complete or partial edentulous patients with both fixed and removable solutions. ${ }^{1}$ Albrektsson et al introduced the concept of foreign body equilibrium, applied to the osseointegration of titanium dental implants. This equilibrium is an immune-mediated foreign body reaction balance during the biological integration of dental implants into the bone. When this equilibrium moves to a disadvantage periimplant bone loss can occur. ${ }^{2}$ Nevertheless, implant failures could still happen in a reduced number of compromised patients, due to the lack of enough understanding of related risk factors.

The causes of bone loss around dental implants and the consequent implant failure may be different and related to implant macro-/microdesign and surface chemical composition, biologic issues, bone quality, surgical technique, host-related factors, and iatrogenic factors.-5 The failure of a dental implant has been classified as early or late depending on its time of occurrence. ${ }^{6}$ Early dental implant failures occur prior to the abutment connection, as consequence of a lack of integration with the bone, ${ }^{5-7}$ and late failures occurs after prosthetic loading, as consequence of plaque-induced peri-implantitis and/or to occlusal overloading. ${ }^{8}$ Osseointegration around titanium implants is a complex biological phenomenon not yet clearly understood. Nevertheless, the surface modifications of titanium dental implants play important roles in the enhancement of osseointegration. With the aim of accelerating and improving the osseointegration process many implant surface treatments were proposed. The surface modification is focused mainly to chemically enhance the roughness of dental implants to increase the appropriate biological response between the living tissues and the dental implants. ${ }^{9,10}$ Furthermore, aside of improving osseointegration, these implant surface modifications have been shown to increase cell viability and biocompatibility. ${ }^{11}$

This topographical change is achieved by acid treatments, sandblasting, or different mechanisms of oxidization. ${ }^{8}$

Sandblasted acid-etched surface (SA) dental implants have a macroroughness achieved with abrasive particles (sandblasting) and micropits obtained by acid etching to improve osseointegration. ${ }^{8,10}$ The SA surface provides an appropriate space for osteoblast adhesion, proliferation, and differentiation. ${ }^{12}$ This result can be further improved by using a double etching process increasing the surface available for new bone ingrowth, hence greatly improving the mechanical fixation. ${ }^{13}$

The more the dental implants used in daily dental practice, the greater the clinical interest becomes in the implants integrating quickly with the bone to be functional. In the last decade, there was a continuous commitment to improve the implant surface to quicken the process of osseointegration and improve its quality. ${ }^{14,15}$ Today, the goal is reducing the healing period from 6 to 8 weeks down to 3 to 4 weeks in all the indications. These efforts have been concentrating in improving the bone to implant interface chemically (by incorporating inorganic phases on or into the titanium oxide layer) or physically (by increasing the level of roughness). ${ }^{16,17}$

Although shorter healing period was presented in many experimental and clinical studies using sandblasted, large grit, and acid-etched (SLA) surfaces, ${ }^{18,19}$ modification of this surface seems to present a stronger bone response than its predecessor. ${ }^{20,21}$

The aim of this split-mouth randomized controlled trial was to compare early implant failure and implant stability of one-stage Hiossen ET III implants with its new hydrophilic (NH) surface, compared with Hiossen ET III implants with the well-known SA surface at 1-year follow-up. The null hypothesis was that there is no difference between groups. The null hypothesis was tested against the alternative hypothesis of differences between them. A preliminary report from one center has been published..$^{10}$ The following trial was reported according to the CONSORT statement guidelines (http:// www.consort-statement.org/).

\section{Materials and Methods}

This study was designed as a split-mouth, randomized controlled trial of parallel groups with two arms and independent outcome assessment when possible, conducted at four centers between November 2017 and May 2018. The protocol was registered in the clinicaltrial.gov (NCT03649100). The 2013 Helsinki declaration was adhered too. The study was performed after approval was received from the Institutional Review Board of the Aldent University, Tirana, Albania (March 2018). All the surgical and prosthetic procedures were performed by one expert clinician at each center.

Any healthy patients, aged 18 years or older, required at least two implants to be rehabilitated with a fixed implant-supported restoration, with a full mouth bleeding and full mouth plaque index $\leq 25 \%$, with a sufficient bone to allow placement of at least 11.5 -mm-long implants, and bone width of at least 6 to $8 \mathrm{~mm}$ for the placement of a regular platform Hiossen ET III implant (Deutsche Osstem GmbH, Eschborn, Germany) were included in this study. The exclusion criteria were in - Table 1.

Patients were informed about the clinical procedures, the materials to be used, the benefits, potential risks and potential complications, as well as any follow-up evaluations required for the clinical study. Patients had to sign the informed consent before including in the study.

A single dose of antibiotic ( $2 \mathrm{~g}$ of amoxicillin and clavulanic acid or clindamycin $600 \mathrm{mg}$ if patients were allergic to penicillin) was administered prophylactically 1 hour before surgery. Patients rinsed with $0.2 \%$ chlorhexidine for 1 minute. Local anesthesia will be induced using a $4 \%$ articaine solution with epinephrine 1:100,000 (Ubistesin; 3M Italia, Milan, Italy). Implants were placed in the planned anatomic sites using a flapless or a mini-flap approach. Bone density was assessed, according to the Lekholm and Zarb classification, during the drilling phase, based on the clinician's experience 
and judgment. Implant site was prepared simultaneously, according to the drilling protocol recommended by the manufacturer (placed at $0.5-\mathrm{mm}$ subcrestal level or deeper according to the bone quality and the soft tissue thickness). The SA surface implants (SA group) or SA surface implants with a newly developed bioabsorbable apatite nanocoating (NH group) were randomized after implant site preparation, immediately before implant placement. Implants used in every group were identical except for the surface treatment. Implants were placed according to a one-stage protocol.

Postsurgical analgesic treatment was performed with ibuprofen $600 \mathrm{mg}$, which was administered twice a day for 2 days after the surgery, and later on, if required. Periapical radiographs were taken with a customized holder at implant placement, at the definitive prosthesis delivery ( - Figs. 1 and $\mathbf{2}$ ), and then yearly ( $\mathbf{- F i g s .} \mathbf{3}$ and $\mathbf{4}$ ). Two to three months after implants placement patients receive single screw-retained restorations.

Table 1 Exclusion criteria

Positive medical findings (such as stroke, recent cardiac infarction, severe bleeding disorder, uncontrolled diabetes, or cancer).

\begin{tabular}{|l|}
\hline Psychiatric therapy. \\
\hline Pregnancy or nursing. \\
\hline Smoking $>10$ cigarettes/d. \\
\hline Insertion torque $<30 \mathrm{Ncm}$. \\
\hline Untreated periodontitis and/or poor oral hygiene. \\
\hline $\begin{array}{l}\text { Acute and chronic infections of the adjacent tissues or natural } \\
\text { dentition. }\end{array}$ \\
\hline $\begin{array}{l}\text { Previous radiotherapy of the oral and maxillofacial region within } \\
\text { the past } 5 \text { y. }\end{array}$ \\
\hline Postextractive implants (at least 3 mo after tooth extraction). \\
\hline Absence of teeth in the opposing jaw. \\
\hline Severe clenching or bruxism. \\
\hline Severe maxillomandibular skeletal discrepancy. \\
\hline
\end{tabular}

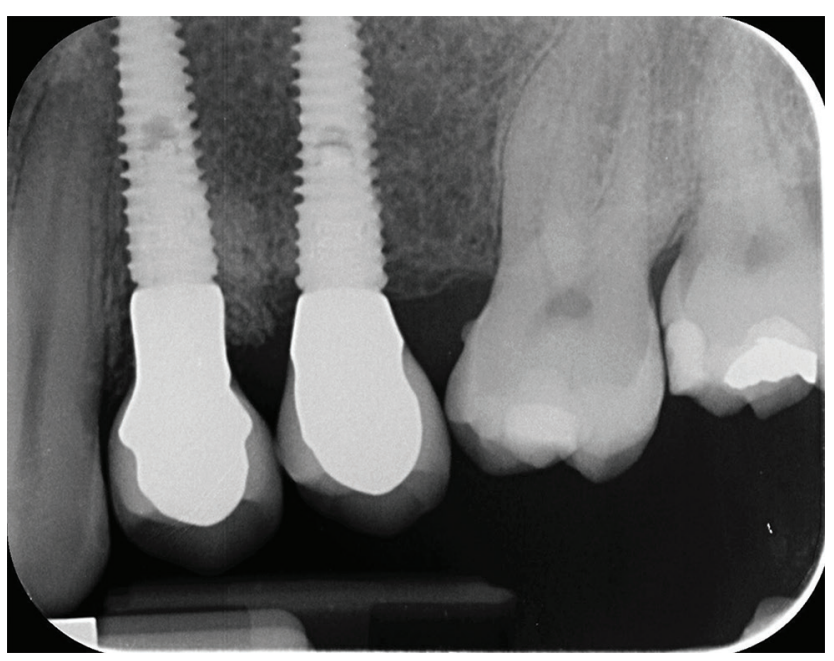

Fig. 1 Periapical radiograph at the definitive prosthesis delivery.
The outcome measures were implant and prosthetic survival rates, any biological or mechanical complications at implants that occurred during the entire observation period, the insertion torque at implant placement, and the implant stability quotient (ISQ).

Success rates of the implants and prostheses were evaluated by an independent assessor (E.X.). An implant was considered a failure if it presented mobility, assessed after the osseointegration period by tapping or rocking the implant head with the metallic handles of two instruments, progressive marginal bone loss or infection, or any mechanical

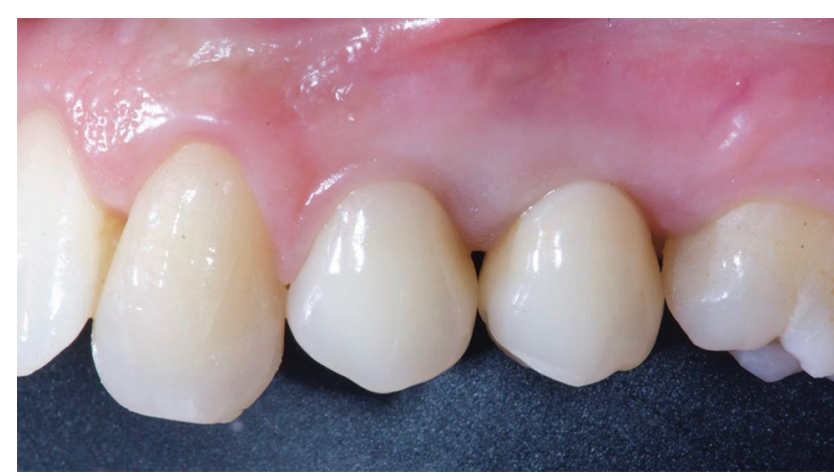

Fig. 2 Clinical picture at the definitive prosthesis delivery.

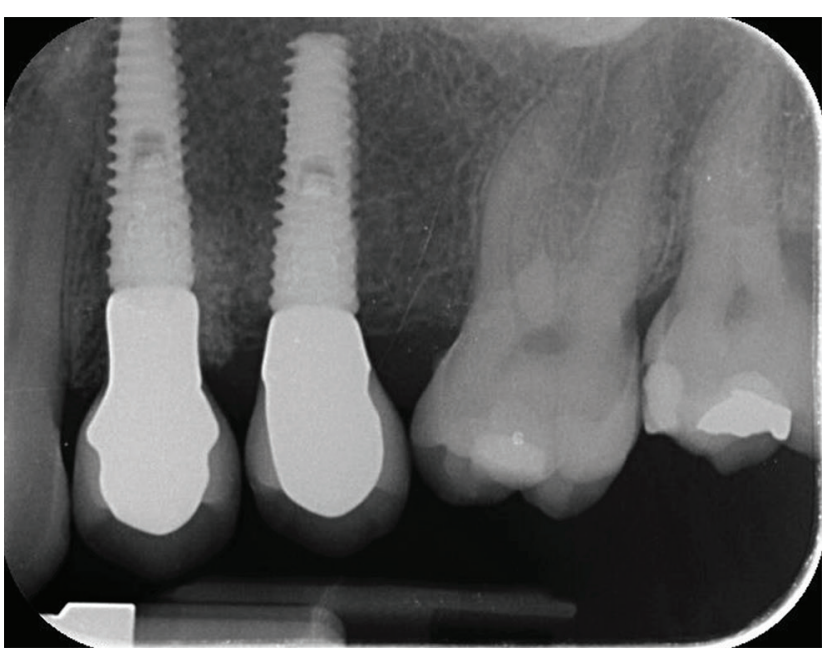

Fig. 3 Periapical radiograph at the 1-year follow-up.

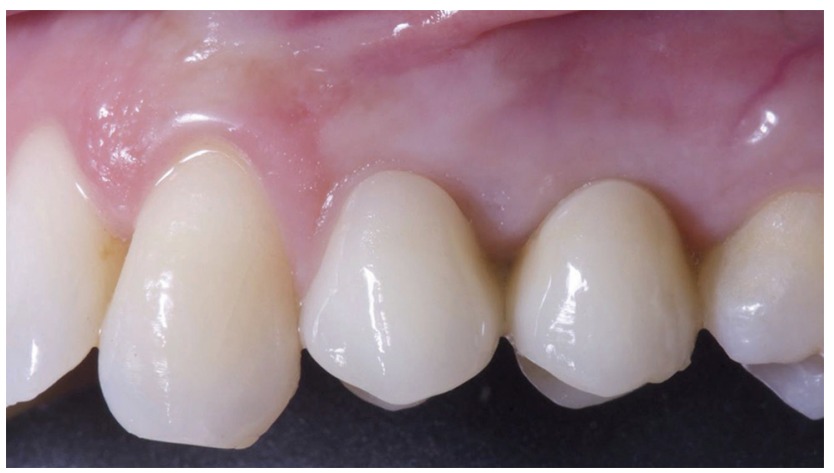

Fig. 4 Clinical picture at the 1-year follow-up. ISQ, implant stability quotient. 
complications rendering the implant unusable, although still mechanically stable in the bone. A prosthesis was considered a failure if it needed to be replaced with another prosthesis.

Biological (pain, swelling, suppuration, etc.) and/or mechanical (screw loosening, fracture of the framework, the veneering material, etc.) complications occurred during the follow-up period. Complications were evaluated and treated by the same surgeon (M.T.).

Insertion torque was recorded at implant placement by the same surgeon (M.T.) using the iChiropro surgical unit (Bien-Air, Bienne, Switzerland).

The insertion torque values of the implants were measured and recorded at implant placement, using the same surgical unit used to place the implants.

The ISQ was measured and recorded using a smart peg (Type 47 cod. 100478, Osstell, Gothenburg, Sweden) connected to the implants, and the Osstell Mentor device (Osstell). Measurements were taken at implant placement, and every week up to 8 weeks after implant placement. In case of ISQ value $<55$ or in case of implant mobility, healing abutment was replaced with a cover screw and the implant was left to heal submerged for at least 6 weeks.

A blind outcome assessor collected the data (E.X.), according to a previously published study. ${ }^{3}$

A pregenerated random list, consisting of a randomized sequence of consecutive numbers matching the two different procedures within group A or group B, was created using random number generator pro 1.91 for Windows (Segobit Software; www.segobit.com). Opaque envelopes containing the randomization codes were sequentially numbered and sealed. According to a pre-generated list, an independent consultant, not previously involved in the trial, prepared all the envelopes and then opened immediately after implant sites preparation. Site one was defined the site with the lower sextant number and the most mesial. Patients and statistician were blinded, while doctor not due to the different opacity of the implant surface. Patient data were collected in an Excel spreadsheet (Microsoft) that reflected the parameters in the patient records. The data were exported into SPSS software for Mac OS X (version 22.0; SPSS, Chicago, Illinois, United States), for the statistical analysis. Descriptive analysis was performed for numeric parameters using means and standard deviations (95\% confidence interval). Complications and failures were compared using the Fisher's exact test. Comparisons between groups (SA vs. $\mathrm{NH}$ ), and between jaws (maxilla vs. mandible) were made by unpaired $t$-test, while the comparison between baseline (T0) and the last follow-up (T8) was made by paired $t$-tests to detect any change during the follow-up. Pearson's correlation coefficient was used to evaluate the correlation between insertion torque at implant placement and ISQ value 8 weeks after implant placement. All statistical comparisons were two-tailed and conducted at the 0.05 level of significance. The patient was used as the statistical unit of analysis.

\section{Results}

Only three out of four centers managed to recruit and treat patients according to the study protocol. Initially, 39 patients were screened but six patients were not included because they did not have sufficient bone to allow placement of 11.5-mm-long and 4-mm diameter implants; two patients were not included because they did not want to participate in the study; and the other two patients were heavy smokers. A total of 29 patients ( 22 females and seven males, with a mean age at implant insertion of $59.9 \pm 11.3$ years) were treated according to the allocated interventions and followed up to 1 year after loading. No patient dropped out. A total of 58 implants (29 with SA surface and 29 with SA surface with the newly developed bioabsorbable apatite nanocoating) were placed. Eighteen patients were rehabilitated in the maxilla and 11 in the mandible. One-year after loading, no implant and no prosthesis failed. Two weeks after implant placement, two Hiossen ET III SA implants showed a small mobility with an ISQ values lower than 55 (49 and 51 , respectively). The healing abutments were replaced with cover screws and the implants were left to heal undisturbed up to 8 weeks after their placement. Nevertheless, no statistically significant difference was reached $(p=0.491)$. In both the implants, the healing abutments were replaced with a cover screw and the implants were left to heal submerged for 6 weeks (up to 8 weeks after implant placement).

The mean insertion torque ranged between 35.0 and $45.0 \mathrm{Ncm}$ (mean of $40.5 \pm 3.23$ [38.17-41.83] Ncm in the SA group and $40.48 \pm 3.49$ [38.02-41.98] $\mathrm{Ncm}$ in the $\mathrm{NH}$ group). The difference between groups was not statistically significant $(p=0.981)$.

The comparison between ISQ values was reported in - Table 2 and - Figs. 5 to 7.

There was a statistically significant difference between groups at the second week after implant placement (T2) with

Table 2 The ISQ values between and within groups

\begin{tabular}{|c|c|c|c|c|c|c|c|c|c|c|}
\hline & T0 $(n=29)$ & $\mathrm{T} 1(n=29)$ & T2 $(n=29)$ & T3 $(n=27)$ & T4 $(n=27)$ & T5 $(n=27)$ & T6 $(n=27)$ & T8 $(n=29)$ & $\begin{array}{l}\text { Difference } \\
\text { T8-T0 }\end{array}$ & $p$-Value \\
\hline SA & \begin{tabular}{|l|}
$77.8 \pm 5.7$ \\
$(75.8-82.2)$
\end{tabular} & \begin{tabular}{|l|}
$76.1 \pm 6.0$ \\
$(74.1-80.9)$
\end{tabular} & \begin{tabular}{|l|}
$72.7 \pm 9.0$ \\
$(68.9-79.1)$
\end{tabular} & \begin{tabular}{|l|}
$75.0 \pm 7.0$ \\
$(71.0-79.0)$
\end{tabular} & \begin{tabular}{|l|}
$77.7 \pm 4.9$ \\
$(75.3-80.7)$
\end{tabular} & \begin{tabular}{|l|}
$78.3 \pm 3.6$ \\
$(75.9-80.1)$
\end{tabular} & \begin{tabular}{|l|}
$78.8 \pm 3.7$ \\
$(76.1-80.4)$
\end{tabular} & \begin{tabular}{|l|}
$78.7 \pm 4.3$ \\
$(76.8-81.7)$
\end{tabular} & $\begin{array}{l}1.0 \pm 4.4 \\
(2.0 \text { to } 3.0)\end{array}$ & 0.266 \\
\hline $\mathrm{NH}$ & \begin{tabular}{|l|}
$76.4 \pm 5.7$ \\
$(71.8-78.2)$
\end{tabular} & \begin{tabular}{|l|}
$77.0 \pm 5.5$ \\
$(71.9-78.1)$
\end{tabular} & \begin{tabular}{|l|}
$76.9 \pm 4.9$ \\
$(72.2-77.8)$
\end{tabular} & $\begin{array}{l}76.9 \pm 4.9 \\
(75.2-80.8)\end{array}$ & $\mid \begin{array}{l}77.1 \pm 4.7 \\
(75.3-80.7)\end{array}$ & \begin{tabular}{|l}
$77.5 \pm 4.2$ \\
$(75.6-80.4)$
\end{tabular} & \begin{tabular}{|l|}
$78.1 \pm 4.3$ \\
$(75.6-80.4)$
\end{tabular} & $\begin{array}{l}78.6 \pm 3.8 \\
(77.8-82.2)\end{array}$ & $\begin{array}{l}1.9 \pm 3.9 \\
(-1.2 \text { to } 3.2)\end{array}$ & $0.019^{a}$ \\
\hline Difference & $\begin{array}{l}1.3 \pm 6.5 \\
(-1.7 \text { to } 5.7)\end{array}$ & $\begin{array}{l}0.8 \pm 5.1 \\
(-1.0 \text { to } 2.9)\end{array}$ & $\begin{array}{l}4.2 \pm 9.1 \\
(-7.2 \text { to } 3.2)\end{array}$ & $\begin{array}{l}1.9 \pm 4.4 \\
(-4.0 \text { to } 1.0)\end{array}$ & $\left|\begin{array}{l}0.5 \pm 4.1 \\
(-3.3 \text { to } 1.3)\end{array}\right|$ & $\begin{array}{l}0.7 \pm 3.1 \\
(-1.7 \text { to } 1.7)\end{array}$ & $\begin{array}{l}0.5 \pm 2.7 \\
(-1.5 \text { to } 1.5)\end{array}$ & $\begin{array}{l}0.1 \pm 3.9 \\
(-2.5 \text { to } 2.0)\end{array}$ & - & - \\
\hline$p$-Value & 0.393 & 0.597 & $0.041^{\mathrm{a}}$ & 0.258 & 0.662 & 0.473 & 0.550 & 0.919 & - & - \\
\hline
\end{tabular}

Abbreviations: ISQ, implant stability quotient; $\mathrm{NH}$, new hydrophilic; SA, sandblasted and acid etched.

aStatistically significant. 

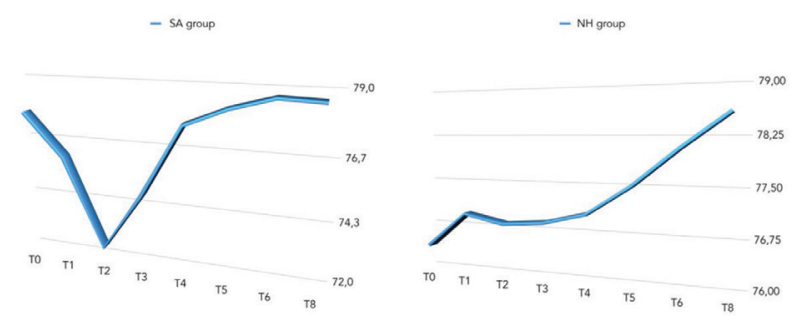

Fig. 5 Overall comparison of mean ISQ values between groups. ISQ, implant stability quotient.

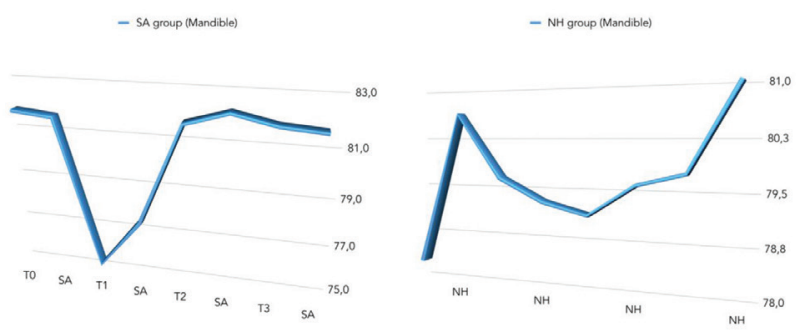

Fig. 6 Comparison of mean ISQ values between groups (in the mandible). ISQ, implant stability quotient.

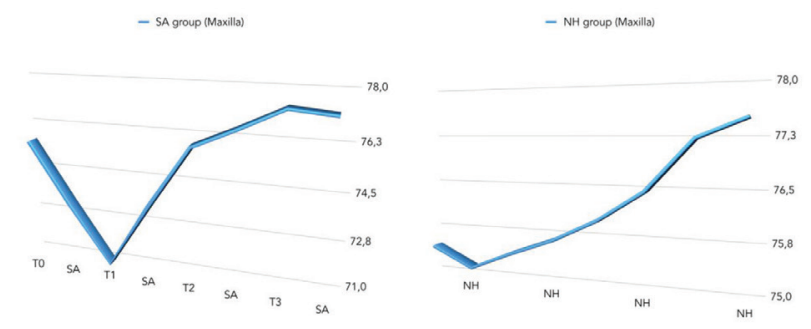

Fig. 7 Comparison of mean ISQ values between groups (in the maxilla). ISQ, implant stability quotient.

higher values in the NH group $(p=0.041)$. Similar results were found in the maxilla ( $p=0.045)$, but not in the mandible $(p=0.362)$. Overall, the ISQ values improved in both groups during the entire follow-up ( 8 weeks), with statistically significant difference in the $\mathrm{NH}$ group $(p=0.019)$, but not in the SA group $(p=0.266)$. A positive correlation was found between initial insertion torque and ISQ with higher value in the $\mathrm{NH}$ group ( 0.73 vs. 0.66 ). Correlation was stronger in the mandible $(\mathrm{SA}=0.71 ; \mathrm{NH}=0.86)$ compared with the maxilla $(\mathrm{SA}=0.52 ; \mathrm{NH}=0.55)$.

\section{Discussion}

Nowadays there is a strong effort in improving the bone to implant interface modifying dental implant surface to improve bone integration and reduce the timing of this process to help clinician in the treatment of edentulous patients.

Exactly in this context our study was oriented, in fact this split-mouth randomized controlled trial was aimed to compare early implant failure and implant stability of one-stage Hiossen ET III implants with its NH surface, compared with
Hiossen ET III implants with the well-known SA surface up to 1-year of follow-up. The null hypothesis of no difference was partially rejected in favor of the alternative hypothesis of difference between groups.

Overall, the mean ISQ improved in both groups during the 8 weeks of follow-up, but the values were with statistically significant difference only in the $\mathrm{NH}$ group $(p=0.019)$. The reason was partial due to the fact that 2 weeks after implants placement the mean ISQ value was statistically significant higher in the $\mathrm{NH}$ group compared with the SA group $(p=0.041)$. A possible explanation could be the unexpected values of ISQ in the SA implant group caused by two implants that showed values $<55$ with slightly implant mobility. As a consequence, implants were left to heal submerged for the next 6 weeks. Nevertheless, it can therefore be assumed that implants with the hydrophilic surface $(\mathrm{NH})$ seem to reduce complications avoiding the ISQ drop back during the remodeling phase allowing accordingly benefits in immediate loading, poor bone quality, post-extractive and maxilla. The main limitations of the present randomized controlled trial are the small sample size and the short-term follow-up. Unfortunately, one center did not participate to the study, contributing to the small sample size.

The ongoing effort of dental companies to improve the interface between bone and implant surface to speed up the process of osseointegration has been proposed by researcher and dental implant companies, and data underlined in this paper, especially for the $\mathrm{NH}$ surface, confirm the chance to reduce time in implant therapy. Today, primary implant stability and absence of micromovements still remain two of the main prerequisites for obtaining a stable osseointegration and the achievement of long-term high-success rates.22,23 On the contrary, if during the first healing period of the implant the primary stability is insufficient, early implant failure can occur. ${ }^{24,25}$

In the present study, similar statistically significance was found in the maxilla $(p=0.045)$, but not in the mandible $(p=0.362)$. To minimize the change of early implant failure, during the last decades it has been suggested that implants should be kept load-free during a healing period of 3 to 4 months in mandibles and 6 to 8 months in maxillae. ${ }^{26}$ Nowadays, the more the implants are used in clinical routine, the greater the clinical interest becomes in the implants integrating quickly with the bone to be functional. An ongoing effort to improve the interface between bone and implant surface to speed up the process of osseointegration has been proposed by researcher and dental implant companies, modifying implant surface roughness and topography. ${ }^{26}$

To better understand dental implant roughness it is commonly divided, depending on the dimension of the measured surface features, into macro-, micro-, and nano roughness. All these kinds of roughness and topography have direct consequences on bone response during the healing period of tissues around dental implants. ${ }^{27-29}$ Nowadays, it is well known that the implant roughness improves osseointegration and the majority of implant types are sandblasted and/ or acidetched to increase their surface texture. ${ }^{24}$ Furthermore the nanometer roughness has the main role in the adsorption of proteins, adhesion of osteoblastic cells, and thus the rate 
of osseointegration..$^{30}$ Furthermore, Schwarz et al showed that hydrophilic surfaces enhance the angiogenesis process when early stages of osseointegration occur. ${ }^{31-33}$ Actually, fast vascularization seems beneficial for bone formation because osteogenic cells have been observed to arise from pericytes adjacent to small blood vessels. ${ }^{23,34}$ In a review of Wennerberg et al, a little clinical evidence was found to clearly state a preference for SLActive over SLA implant.. ${ }^{35,36}$ At 1-year follow-up, there was a high survival rate $(100 \%$ for SLActive vs. $96 \%$ for SLA implants) and low crestal bone loss $<0.4 \mathrm{~mm}$ in both groups with no significant difference.

Recent literature suggest that an optimal insertion torque could be around $30 \mathrm{Ncm}$ to obtain a successful and durable osseointegration, which is also sufficient to allow both conventional and immediate occlusal loading of dental implants. ${ }^{37}$ The reason why dental implant companies and researchers are focused on implant design improvements and surface modification is to help both clinicians and patients to fasten implant surgery and prosthetic timing, ${ }^{26,38-44}$ for same reasons, researchers are focused on digital dentistry and in developing guidelines in implant dentistry. ${ }^{45-47}$

In the present study, a positive correlation was found between initial insertion torque and ISQ with higher value in the $\mathrm{NH}$ group $(0.73$ vs. 0.66$)$. Correlation was stronger in the mandible $(\mathrm{SA}=0.71 ; \mathrm{NH}=0.86$ ) compared with the maxilla $(\mathrm{SA}=0.52 ; \mathrm{NH}=0.55)$. The clinical implication from this randomized controlled trial may be that implants with NH surface modification could be an important option when treating patients, especially when the timing of loading is crucial. Considering the positive correlation found between high torque insertion and an increased ISQ level, and better results in the maxilla, this surface treatment could be a viable treatment option in case of immediate loading, poor bone quality (such us posterior maxilla), postextractive implants, or high risks patients, such us immunocompromised patients or heavy smokers.

\section{Conclusions}

Considering the limitation of this multicenter, split-mouth, randomized controlled trial, $\mathrm{NH}$ implants are a viable alternative to SA surface, as they seem to avoid the ISQ drop during the remodeling phase. It can be beneficial in immediate loading, poor bone quality, postextractive implants, smoking, and immunosuppression. Further studies are needed to improve the number of patients and long-term follow-up.

\section{Ethical Approval}

The study performed after approval was received from the Institutional Review Board of the Aldent University, Tirana, Albania (March 2018). Informed consent (written) was obtained from all patients before the samples were definitively enrolled.

\section{Consent for Publication}

The patients signed an informed consent form agreeing with the publication of this study.

\section{Availability of Data and Material}

The datasets used during the current study are available from supplementary file.

\section{Authors' Contributions}

M.T. performed all the cases at center 1, contributed to the conception of the work and to the acquisition of the data, and finally given final approval of the version to be published. M.M. and E.X. drafted the manuscript and gave their final approval of the version to be published. N.B. and F.G. performed all the cases at centers 2 and 3, respectively. S.M.M.revisited the manuscript critically for important intellectual content. L.A.I. contributed to the analysis of the data and revisited the manuscript critically.

\section{Funding}

This publication was supported by University funding (L.A.I.): grant FAR2019.

\section{Conflict of Interest}

None declared.

\section{References}

1 Albrektsson T, Chrcanovic B, Östman P-O, Sennerby L. Initial and long-term crestal bone responses to modern dental implants. Periodontol 2000 2017;73(1):41-50

2 Trindade R, Albrektsson T, Tengvall P, Wennerberg A. Foreign body reaction to biomaterials: on mechanisms for buildup and breakdown of osseointegration. Clin Implant Dent Relat Res 2016;18(1):192-203

3 Albrektsson T, Canullo L, Cochran D, De Bruyn $\mathrm{H}$. "Peri-implantitis": a complication of a foreign body or a man-made "disease". Facts and fiction. Clin Implant Dent Relat Res 2016;18(4):840-849

4 Albrektsson T, Dahlin C, Jemt T, Sennerby L, Turri A, Wennerberg A. Is marginal bone loss around oral implants the result of a provoked foreign body reaction? Clin Implant Dent Relat Res 2014;16(2):155-165

5 Albrektsson T, Donos N; Working Group 1. Implant survival and complications. The Third EAO consensus conference 2012. Clin Oral Implants Res 2012;23(suppl 6):63-65

6 Cicciu M, Bramanti E, Matacena G, Guglielmino E, Risitano G. FEM evaluation of cemented-retained versus screw-retained dental implant single-tooth crown prosthesis. Int J Clin Exp Med 2014;7(4):817-825

7 Bramanti E, Cervino G, Lauritano F, et al. FEM and Von Mises analysis on prosthetic crowns structural elements: evaluation of different applied materials. ScientificWorldJournal 2017;2017:1029574

8 Anil S, Anand PS, Alghamdi H, Jansen JA, Dental implant surface enhancement and osseointegration. In: Turkyilmez I, ed. Implant Dentistry-A Rapidly Evolving Practice. Rijeka, Croatia: InTech; 2011 83-87

9 Cicciù M, Risitano G, Maiorana C, Franceschini G. Parametric analysis of the strength in the "Toronto" osseous-prosthesis system. Minerva Stomatol 2009;58(1-2):9-23

10 Tallarico M, Baldini N, Martinolli M, et al. Do the new hydrophilic surface have any influence on early success rate and implant stability during osseointegration period? Four-month preliminary results from a split-mouth, randomized controlled trial. Eur J Dent 2019;13(1):95-101

11 Tallarico M, Vaccarella A, Marzi GC. Clinical and radiological outcomes of 1-versus 2-stage implant placement: 1-year results of a randomised clinical trial. Eur J Oral Implantology 2011;4(1):13-20 
12 Le Guéhennec L, Soueidan A, Layrolle P, Amouriq Y. Surface treatments of titanium dental implants for rapid osseointegration. Dent Mater 2007;23(7):844-854

13 Chrcanovic BR, Albrektsson T, Wennerberg A. Reasons for failures of oral implants. J Oral Rehabil 2014;41(6):443-476

14 Renvert S, Polyzois I, Claffey N. How do implant surface characteristics influence peri-implant disease? J Clin Periodontol 2011;38(suppl 11):214-222

15 Dohan Ehrenfest DM, Coelho PG, Kang BS, Sul YT, Albrektsson T. Classification of osseointegrated implant surfaces: materials, chemistry and topography. Trends Biotechnol 2010;28(4): 198-206

16 Xuereb M, Camilleri J, Attard NJ. Systematic review of current dental implant coating materials and novel coating techniques. Int J Prosthodont 2015;28(1):51-59

17 Piattelli A, Cosci F, Scarano A, Trisi P. Localized chronic suppurative bone infection as a sequel of peri-implantitis in a hydroxyapatite-coated dental implant. Biomaterials 1995;16(12): 917-920

18 Payne AG, Tawse-Smith A, Duncan WD, Kumara R. Conventional and early loading of unsplinted ITI implants supporting mandibular overdentures. Clin Oral Implants Res 2002;13(6): 603-609

19 Cochran DL, Schenk RK, Lussi A, Higginbottom FL, Buser D. Bone response to unloaded and loaded titanium implants with a sandblasted and acid-etched surface: a histometric study in the canine mandible. J Biomed Mater Res 1998;40(1):1-11

20 Khandelwal N, Oates TW, Vargas A. Alexander PP, Schoolfield JD, Alex McMahan C. Conventional SLA and chemically modified SLA implants in patients with poorly controlled type 2 diabetes mellitus-a randomized controlled trial. Clin Oral Implants Res 2013;24(1):13-19

21 Brånemark PI, Hansson $\mathrm{BO}$, Adell R, et al. Osseointegrated implants in the treatment of the edentulous jaw. Experience from a 10-year period. Scand J Plast Reconstr Surg Suppl 1977; $16: 1-132$

22 Makary C, Rebaudi A, Sammartino G, Naaman N. Implant primary stability determined by resonance frequency analysis: correlation with insertion torque, histologic bone volume, and torsional stability at 6 weeks. Implant Dent 2012;21(6):474-480

23 Szmukler-Moncler S, Salama H, Reingewirtz Y, Dubruille JH. Timing of loading and effect of micromotion on bone-dental implant interface: review of experimental literature. J Biomed Mater Res 1998;43(2):192-203

24 Lioubavina-Hack N, Lang NP, Karring T. Significance of primary stability for osseointegration of dental implants. Clin Oral Implants Res 2006;17(3):244-250

25 Brånemark PI, Adell R, Breine U, Hansson BO, Lindström J, Ohlsson A. Intra-osseous anchorage of dental prostheses. I. Experimental studies. Scand J Plast Reconstr Surg 1969;3(2):81-100

26 Tallarico M, Vaccarella A, Marzi GC, Alviani A, Campana V. A prospective case-control clinical trial comparing 1- and 2-stage Nobel Biocare TiUnite implants: resonance frequency analysis assessed by Osstell Mentor during integration. Quintessence Int 2011;42(8):635-644

27 Junker R, Dimakis A, Thoneick M, Jansen JA. Effects of implant surface coatings and composition on bone integration: a systematic review. Clin Oral Implants Res 2009;20(suppl 4): 185-206

28 Rupp F, Liang L, Geis-Gerstorfer J. Scheideler L, Hüttig F. Surface characteristics of dental implants: a review. Dent Mater 2018;34(1):40-57

29 Heitz-Mayfield LJ. Peri-implant diseases: diagnosis and risk indicators. J Clin Periodontol 2008;35(suppl 8):292-304

30 Braceras I, Alava JI, Oñate JI, et al. Improved osseointegration in ion implantation-treated dental implants. Surf Coat Tech 2002;158-159:28-32
31 Schwarz F, Herten M, Sager M, Wieland M, Dard M, Becker J. Histological and immunohistochemical analysis of initial and early osseous integration at chemically modified and conventional SLA titanium implants: preliminary results of a pilot study in dogs. Clin Oral Implants Res 2007;18(4):481-488

32 Long MW, Robinson JA, Ashcraft EA, Mann KG. Regulation of human bone marrow-derived osteoprogenitor cells by osteogenic growth factors. J Clin Invest 1995;95(2):881-887

33 Reilly TM, Seldes R, Luchetti W, Brighton CT. Similarities in the phenotypic expression of pericytes and bone cells. Clin Orthop Relat Res 1998;(346):95-103

34 Murphy M, Walczak MS, Thomas AG, Silikas N, Berner S, Lindsay R. Toward optimizing dental implant performance: surface characterization of Ti and TiZr implant materials. Dent Mater 2017;33(1):43-53

35 Wennerberg A, Galli S, Albrektsson T. Current knowledge about the hydrophilic and nanostructured SLActive surface. Clin Cosmet Investig Dent 2011;3:59-67

36 Smeets R, Stadlinger B, Schwarz F, et al. Impact of dental implant surface modifications on osseointegration. BioMed Res Int 2016;2016:6285620

37 Faot F, Bielemann AM, Schuster AJ, et al. Influence of insertion torque on clinical and biological outcomes before and after loading of mandibular implant-retained overdentures in atrophic edentulous mandibles. BioMed Res Int 2019;2019: 8132520

38 Herford AS, Miller M, Lauritano F, Cervino G, Signorino F, Maiorana $C$. The use of virtual surgical planning and navigation in the treatment of orbital trauma. Chin J Traumatol 2017;20(1):9-13

39 Stacchi C, Lombardi T, Cusimano P, et al. Bone scrapers versus piezoelectric surgery in the lateral antrostomy for sinus floor elevation. J Craniofac Surg 2017;28(5):1191-1196

40 Lo Giudice G, Cicciù M, Cervino G, Lizio A, Visco AM. Flowable resin and marginal gap on tooth third medial cavity involving enamel and radicular cementum: a SEM evaluation of two restoration techniques. Indian J Dent Res 2012;23(6): 763-769

41 Cervino G, Terranova A, Briguglio F, et al. Diabetes: oral health related quality of life and oral alterations. BioMed Res Int 2019;2019:5907195

42 Lo Giudice G, Lipari F, Lizio A, Cervino G, Cicciù M. Tooth fragment reattachment technique on a pluri traumatized tooth. J Conserv Dent 2012;15(1):80-83

43 Cervino G, Fiorillo L, Herford AS, et al. Molecular biomarkers related to oral carcinoma: clinical trial outcome evaluation in a literature review. Dis Markers 2019;2019:8040361

44 Pozzi A, Tallarico M, Moy PK. Immediate loading with a novel implant featured by variable-threaded geometry, internal conical connection and platform shifting: three-year results from a prospective cohort study. Eur J Oral Implantol 2015;8(1):51-63

45 Cervino G, Fiorillo L, Arzukanyan AV, Spagnuolo G, Cicciù M. Dental restorative digital workflow: digital smile design from aesthetic to function. Dent J (Basel) 2019;7(2):28

46 Lavorgna L, Cervino G, Fiorillo L, et al. Reliability of a virtual prosthodontic project realized through a $2 \mathrm{~d}$ and $3 \mathrm{~d}$ photographic acquisition: an experimental study on the accuracy of different digital systems. Int J Environ Res Public Health 2019;16(24):5139

47 Laino L, Cicciù M, Fiorillo L, et al. Surgical risk on patients with coagulopathies: guidelines on hemophiliac patients for oro-maxillofacial surgery. Int J Environ Res Public Health 2019; 16(8):1386 